\title{
The cumulative incidence and trends of rare diseases in South Korea: a nationwide study of the administrative data from the National Health Insurance Service database from 2011-2015
}

Sung-Shil Lim ${ }^{1,2}$, Wanhyung Lee ${ }^{3}$, Yeong-Kwang Kim²,2, Jihyun Kim,2, Jong Heon Park ${ }^{4}$, Bo Ram Park ${ }^{4}$ and Jin-Ha Yoon ${ }^{2,5^{*}}$ (D)

\begin{abstract}
Background: The burden of rare diseases on society and patients' families has increased in Korea. However, because of the infrequency of rare diseases, there is a lack of resources and information to address these cases and inadequate funding for the management of these patients. We investigated the average annual cumulative incidence of rare diseases and the trends in annual cumulative incidence from 2011 to 2015 in Korea by using nationwide administrative data from the Korean National Health Insurance Service (NHIS) database for patients registered with the co-payment assistance policy for rare and incurable diseases. Annual cumulative incidence per 10,000,000 was calculated as the total number of newly enrolled patients with the Korean Standard Classification of Diseases (KCD)-7 code in the register, divided by the number of residents with health insurance coverage during each year. We employed simple linear regression analysis to evaluate the trends in annual cumulative incidence/10,000,000 population per year for each rare disease.

Results: Overall, national support was provided for patients with 415 KCD codes listed among the targeted rare diseases. The total number of newly enrolled patients with rare diseases was 53,831 in 2011, 52,658 in 2012, 52,955 in 2013, 71,530 in 2014, and 70,559 in 2015. The number of rare diseases with an average annual cumulative incidence of 100/10,000,000 and above was 22 (5.30\%), while there were 227 (54.70\%) and 148 (35.66\%) with an average cumulative incidence between $1 / 10,000,000$ and $100 / 10,000,000$ and less than $1 / 10,000,000$, respectively. The trends in the annual cumulative incidence for 43 rare diseases were statistically significant ( $p$-value $<0.05$ ). The rare diseases for which the incremental trend per year was statistically significant were sarcoidosis (D86, D86.0, D86.1, D86.2, D86.3, D86.8, D86.9), Parkinson's disease (G20), Guillain-Barré syndrome (G61.0), primary biliary cirrhosis (K74.3) and Sjogren's syndrome (M35.0).

Conclusions: The number of rare diseases showing an increasing trend in annual cumulative incidence was higher than the number of diseases showing a decreasing trend in annual cumulative incidence. Given that the definition and diagnosis vary based on country and that there is difficulty in identifying valid cases, further detection strategies are needed to establish the incidence of each rare disease considering the importance of establishing a health policy based on the actual incidence of the targeted diseases.
\end{abstract}

Keywords: Rare disease, Incidence, Administrative data, Population-based

\footnotetext{
* Correspondence: flyinyou@yuhs.ac

${ }^{2}$ The Institute for Occupational Health, Yonsei University College of Medicine,

Seoul, Republic of Korea

${ }^{5}$ Department of Preventive Medicine and Public Health, Yonsei University

College of Medicine, Seoul, Republic of Korea

Full list of author information is available at the end of the article
}

(c) The Author(s). 2019 Open Access This article is distributed under the terms of the Creative Commons Attribution 4.0 International License (http://creativecommons.org/licenses/by/4.0/), which permits unrestricted use, distribution, and reproduction in any medium, provided you give appropriate credit to the original author(s) and the source, provide a link to the Creative Commons license, and indicate if changes were made. The Creative Commons Public Domain Dedication waiver (http://creativecommons.org/publicdomain/zero/1.0/) applies to the data made available in this article, unless otherwise stated. 


\section{Background}

A rare disease is defined as any disease with a low prevalence. However, the exact definitions of rare diseases vary from country to country. In the European Union, a rare disease is defined as one that affects less than 1 in 2000 [1]. A disease is defined as rare in the United States of America when it affects fewer than 200,000 people at any given time [2]. In South Korea, the term rare disease applies to diseases for which there are fewer than 20,000 patients, or for which the prevalence is unknown owing to difficulties in diagnosing the disease or that are designated by the procedures and standards set by the Ministry of Health and Welfare [3]. Despite the low prevalence of each rare disease, the burden of rare diseases on patients' families and society is considerable owing to the chronicity and relatively incurable pattern of rare diseases [4]. According to the report from the Commission of the European Communities in 2008, it is estimated that 30 million European Union citizens have rare diseases [5]. In South Korea, the patient cost of care for rare diseases has increased. The total cost of medical care benefits for rare and intractable diseases increased from 2.6 billion US dollars in 2010 to 4.1 billion US dollars in 2016 [6]. Due to the chronicity of several rare diseases and the high economic cost of treatment, patients with rare diseases need social support. However, considering the infrequency of rare diseases, affected patients are likely to be neglected, resulting in a lack of resources and information to address these cases and inadequate funding for the proper care of these patients [7]. Furthermore, many patients with rare diseases would have visited several clinics or hospitals, but would only receive symptomatic treatment, owing to the lack of knowledge about the pathophysiology of rare diseases and the difficulties in their proper diagnoses [8-11]. After being correctly diagnosed with a rare disease, affected patients may not receive proper care because of the lack of adequate treatment or high price of medication ("orphan drugs") [4, 12, 13].

South Korea introduced a legislation regarding orphan drugs in 2003 and regarding the management of rare diseases in 2016 [3, 14]. Even before the introduction of the rare disease management act, the government had subsidized medical expenses for patients with rare and intractable diseases through a co-payment assistance policy that was in place since 2001. The targeted rare diseases covered by the policy (see Additional file 1) are determined via expert consultation based on their rarity and severity. In order to benefit from national support, the disease must be registered in the register of the co-payment assistance policy, which is supported by the National Health Insurance Service (NHIS). The entire population residing within the territory of Korea is covered by the National Health Insurance (NHI) of Korea [15]. Registered patients with rare diseases make out-of-pocket payments that comprise about $10 \%$ of the total cost of medical treatment, which is normally $20-60 \%$ of the total cost of treatment. Furthermore, patients with some rare diseases receive support for the purchase of assistive devices such as those for the disabled, respiratory aids, and cough inducers, as well as support towards the cost of caregiving [3].

The investigation of the incidence and prevalence of diseases should be preceded by establishing the public health policy for the specific disease. However, investigators have faced difficulties in studying the epidemiology of rare diseases because of difficulties in detecting the few existing cases [16]. Several studies have attempted to understand the epidemiology of rare diseases. For example, Seo et al., using NHIS data, reported that the prevalence of amyloidosis was 0.93 (95\% confidence interval [CI]: 0.81-1.04) in Korea [17]. Ryder et al. reported that the point prevalence of Duchenne muscular dystrophy per 100,000 men in France, the USA, UK, and Canada was 10.9, 1.9, 2.2, and 6.1, respectively [18]. Groth et al. showed that the annual median incidence of Marfan syndrome was 0.19/100,000 population (range: 0.0-0.7) [19]. Furthermore, Orphanet conducted a systemic review of literature to estimate the incidence and prevalence of rare diseases [20]. However, most of the studies did not use the rare disease register data, but rather the medical claim data $[17,18]$; thus, it is not easy to obtain the real incidence of each rare disease [21]. Therefore, population-based administrative records of rare disease registries are appropriate sources of data to conduct a passive surveillance of rare diseases to maximize valid case detection [21].

To investigate the population-based annual cumulative incidence (2011-2015) of rare diseases in Korea according to the Korean Standard Classification of Diseases (KCD) [22], we used the administrative data from the register of the co-payment assistance policy maintained by the NHIS for rare and incurable diseases in South Korea. We also examined the trends in the annual cumulative incidence of each rare disease from 2011 to 2015.

\section{Methods \\ Study population}

We used the administrative data regarding application for registration in the co-payment assistance policy with the NHIS for the period of 2011-2015 in order to determine the population-based incidence of each rare disease in Korea. These data included the information of the applicants, code of the targeted disease classified per the KCD-7 based on the International Classification of Diseases (ICD)-10 [23], date of definite diagnosis and start date of the application for co-payment assistance, and tests performed for the diagnosis of the rare disease. We included all patients who registered for the co-payment assistance policy and actually received national support. Only patients who met the diagnostic criteria which NHIS 
had defined for each rare disease on the basis of the results of comprehensive medical tests including imaging studies, biochemistry, immunology, smear, culture test, histological examination and clinical diagnosis by the physician could be registered in the system. For example, only patients with Crohn's disease confirmed by the combined results of imaging studies, clinical evaluation, endoscopy and biopsy are eligible for national support by NHIS. The annual cumulative incidence of rare diseases was calculated using the KCD code in the application. Overall, $415 \mathrm{KCD}$ codes were used to calculate the annual cumulative incidence of each rare disease. We used the year of definite diagnosis by the physician in the application form as the year of disease occurrence. We excluded the data regarding reapplications to extend national support, which occurs every 5 years, thus including data for only the first enrolment for each disease. Finally, we excluded patients with missing values for sex and age.

\section{Statistical analysis}

All residents within the territory of Korea are covered by the NHI of Korea [15]. It is mandatory for all citizens of Korea to be associated with the NHI by law. The number of residents with health insurance coverage was $50,908,646$ in $2011,51,169,141$ in $2012,51,448,491$ in $2013,51,757,146$ in 2014, and 52,034,424 in 2015, which represent approximately $98 \%$ of the people living in the territory of Korea [24].

Annual cumulative incidence per 10,000,000 was calculated as the total number of newly enrolled patients with the KCD-7 code in the register of the co-payment assistance policy for rare and incurable diseases during a calendar year, divided by the number of residents with health insurance coverage in each year (see Additional file 2). The average annual cumulative incidence was calculated as the mean annual cumulative incidence from 2011 to 2015 . However, we calculated the average annual cumulative incidence during the 2 years (2014-2015) of newly enrolled targeted diseases (KCD codes: G40.4, G40.40, G40.41) owing to the expansion of coverage for these diseases in 2014. We employed a simple linear regression analysis to evaluate the trends in annual cumulative incidence/10,000,000 population per year for each rare disease. $P$-values $<0.05$ were considered statistically significant. All analyses were conducted using SAS version 9.4 (SAS Institute, Cary, NC, USA) and R (R Development Core Team, Vienna, Austria).

\section{Results}

The total number of newly enrolled patients with rare diseases benefiting from national support was 53,831 in $2011,52,658$ in 2012, 52,955 in 2013, 71,530 in 2014, and 70,559 in 2015. Table 1 shows the average annual cumulative incidence per $10,000,000$ of the insured population
(2011-2015) with rare diseases and the trends in annual cumulative incidence per year for each rare disease in the register of the co-payment assistance according to the $\mathrm{KCD}$ code. The number of rare diseases with an average annual cumulative incidence of 100/10,000,000 and above was 22 (5.30\%); the number of rare diseases with an average annual cumulative incidence between $1 / 10,000,000$ and 100/10,000,000 was $227(54.70 \%)$ and that for rare diseases with an average annual cumulative incidence less than $1 / 10,000,000$ was 148 (35.66\%) (data not shown in table). There were no reported cases of 18 (4.34\%) KCD code diseases during the study period.

The trends in annual cumulative incidence for 43 rare diseases were statistically significant $(p$-value $<0.05)$. Among them, 34 rare diseases including sarcoidosis (D86.0, D86.1, D86.2), Parkinson's disease (G20), Guillain-Barré syndrome (G61.0), idiopathic pulmonary fibrosis (J84.18), primary biliary cirrhosis (K74.3), autoimmune hepatitis (K75.4), bullous pemphigoid (L12.0), microscopic polyangiitis (M31.7) and Sjogren's syndrome (M35.0) showed an increasing trend and 9 rare diseases including neutropenia (D70), nephrogenic diabetes insipidus (N25.1) and tuberous sclerosis (Q85.1) showed a decreasing trend. The rare diseases, including those with the top 10 highest average annual cumulative incidences and for which increment trends per year were statistically significant were Parkinson's disease (G20; $\beta=140.88$; $p$-value $=0.029)$, dilated cardiomyopathy, congestive cardiomyopathy (I42.0; $\beta=44.36 ; p$-value $=0.037$ ), respiratory distress syndrome of the newborn (P22.0; $\beta=85.09$; $p$-value $=0.001)$, and idiopathic pulmonary fibrosis $(\mathrm{J} 84.18$; $\beta=19.79 ; p$-value $=0.006$ ).

\section{Discussion}

This study determined the annual cumulative incidence of rare diseases in Korea using nationwide data obtained from the administrative register for the co-payment assistance policy for rare and incurable diseases in the Korean NHIS from 2011 to 2015. Our results showed that the number of rare diseases showing an increasing trend in average annual cumulative incidence was higher than the number of diseases showing a decreasing trend in average annual cumulative incidence (34 [8.19\%] vs. 4 [0.96\%]).

The rare disease with the highest average annual cumulative incidences and for which the increment trends per year were statistically significant was Parkinson's disease. Parkinson's disease was the most common rare disease (average annual cumulative incidence: 2066.43/10,000,000) and its annual cumulative incidence increased from 2011 to 2015 in our study. The annual cumulative incidence of Parkinson's disease per $10,000,000$ was 1884.36 in 2011, 1882.58 in 2012, 1945.05 in 2013, 2164.53 in 2014 and 2447.80 in 2015 (see Additional file 2). Parkinson's disease 
Table 1 Average annual cumulative incidence/10,000,000 and trend of annual cumulative incidence of rare diseases in the register of the co-payment assistance policy according to KCD codes from 2011 to 2015

\begin{tabular}{|c|c|c|c|}
\hline $\mathrm{KCD}$ & Average annual cumulative incidence ${ }^{a}$ & Annual trend $(\beta)^{\mathrm{b}}$ & $p$-value \\
\hline A81 & 0.31 & -0.02 & 0.898 \\
\hline A81.0 & 6.48 & 0.71 & 0.203 \\
\hline A81.1 & 0.04 & 0.04 & 0.182 \\
\hline A81.2 & 0.74 & -0.16 & 0.063 \\
\hline A81.8 & 0.08 & 0.02 & 0.646 \\
\hline A81.9 & 0.12 & 0.02 & 0.654 \\
\hline B45 & 0.35 & 0.08 & 0.322 \\
\hline B45.0 & 7.46 & 0.37 & 0.353 \\
\hline B45.1 & 3.69 & -0.10 & 0.736 \\
\hline B45.2 & 0.16 & -0.08 & 0.138 \\
\hline B45.3 & 0.08 & -0.02 & 0.629 \\
\hline B45.7 & 0.15 & 0.08 & 0.141 \\
\hline B45.8 & 0.15 & 0.08 & 0.142 \\
\hline B45.9 & 1.16 & 0.23 & 0.102 \\
\hline D35.2 & 562.81 & 12.94 & 0.366 \\
\hline D55.0 & 0.86 & -0.24 & 0.268 \\
\hline D55.2 & 0.19 & 0.02 & 0.818 \\
\hline D56 & 0.00 & . & \\
\hline D56.0 & 0.08 & 0.02 & 0.639 \\
\hline D56.1 & 1.86 & 0.16 & 0.491 \\
\hline D56.3 & 0.19 & -0.02 & 0.704 \\
\hline D56.4 & 0.08 & 0.02 & 0.639 \\
\hline D56.8 & 0.15 & 0.08 & 0.142 \\
\hline D56.9 & 1.24 & 0.19 & 0.430 \\
\hline D59.5 & 5.82 & 0.90 & 0.107 \\
\hline D60 & 0.35 & 0.09 & 0.366 \\
\hline D60.0 & 0.19 & -0.06 & 0.211 \\
\hline D60.1 & 0.12 & 0.02 & 0.647 \\
\hline D60.8 & 0.62 & 0.09 & 0.701 \\
\hline D60.9 & 5.86 & 0.54 & 0.403 \\
\hline D61.0 & 3.74 & -0.43 & 0.133 \\
\hline D61.2 & 0.58 & 0.10 & 0.487 \\
\hline D61.3 & 18.52 & 1.48 & 0.090 \\
\hline D61.8 & 2.53 & -0.05 & 0.803 \\
\hline D61.9 & 123.60 & 1.69 & 0.612 \\
\hline D64.4 & 0.08 & -0.02 & 0.629 \\
\hline D69.1 & 3.32 & -1.17 & 0.143 \\
\hline D69.30 & 5.60 & -0.21 & 0.777 \\
\hline D69.6 & 99.82 & $-8.77^{*}$ & 0.046 \\
\hline D70 & 41.74 & $-3.29^{*}$ & 0.044 \\
\hline D71 & 1.72 & -0.48 & 0.236 \\
\hline D76.1 & 21.40 & $1.36^{*}$ & 0.012 \\
\hline D76.3 & 9.39 & 1.03 & 0.374 \\
\hline
\end{tabular}


Table 1 Average annual cumulative incidence/10,000,000 and trend of annual cumulative incidence of rare diseases in the register of the co-payment assistance policy according to KCD codes from 2011 to 2015 (Continued)

\begin{tabular}{|c|c|c|c|}
\hline $\mathrm{KCD}$ & Average annual cumulative incidence ${ }^{a}$ & Annual trend $(\beta)^{\mathrm{b}}$ & $p$-value \\
\hline $\mathrm{D} 80$ & 0.16 & -0.08 & 0.305 \\
\hline D80.0 & 1.01 & 0.33 & 0.465 \\
\hline D80.1 & 1.40 & 0.17 & 0.740 \\
\hline D80.2 & 1.64 & -0.34 & 0.304 \\
\hline D80.3 & 5.11 & $1.42^{*}$ & 0.033 \\
\hline D80.4 & 0.04 & 0.04 & 0.182 \\
\hline D80.5 & 0.12 & 0.06 & 0.559 \\
\hline D80.6 & 0.04 & -0.02 & 0.559 \\
\hline D80.7 & 0.08 & 0.08 & 0.182 \\
\hline D80.8 & 0.08 & 0.00 & 0.984 \\
\hline D80.9 & 0.23 & 0.04 & 0.647 \\
\hline D81 & 0.00 & & . \\
\hline D81.1 & 0.08 & 0.04 & 0.311 \\
\hline D81.2 & 0.04 & 0.02 & 0.559 \\
\hline D81.8 & 0.04 & -0.04 & 0.182 \\
\hline D81.9 & 0.23 & 0.00 & 0.984 \\
\hline D82 & 0.00 & . & . \\
\hline D82.0 & 0.31 & 0.04 & 0.660 \\
\hline D82.1 & 2.13 & 0.65 & 0.140 \\
\hline D82.3 & 0.08 & 0.00 & 0.984 \\
\hline D82.4 & 0.31 & -0.08 & 0.322 \\
\hline D82.8 & 0.04 & 0.04 & 0.182 \\
\hline D83 & 0.00 & . & \\
\hline D83.0 & 0.12 & 0.00 & 0.996 \\
\hline D83.9 & 0.31 & 0.06 & 0.499 \\
\hline D84 & 0.04 & 0.02 & 0.559 \\
\hline D84.0 & 0.00 & . & \\
\hline D84.1 & 1.32 & -0.07 & 0.752 \\
\hline D84.8 & 0.08 & 0.00 & 0.984 \\
\hline D84.9 & 0.78 & -0.04 & 0.568 \\
\hline D86 & 3.81 & -0.35 & 0.282 \\
\hline D86.0 & 22.89 & $3.72^{*}$ & 0.003 \\
\hline D86.1 & 12.46 & $1.64^{*}$ & 0.027 \\
\hline D86.2 & 8.96 & $1.35^{*}$ & 0.017 \\
\hline D86.3 & 4.61 & 0.87 & 0.219 \\
\hline D86.8 & 4.04 & -0.06 & 0.885 \\
\hline D86.9 & 26.68 & 1.56 & 0.133 \\
\hline E22.0 & 28.45 & 3.00 & 0.318 \\
\hline E23.0 & 35.21 & -0.14 & 0.958 \\
\hline E24.0 & 3.84 & 0.48 & 0.508 \\
\hline E24.1 & 0.08 & 0.00 & 0.984 \\
\hline E24.3 & 0.16 & -0.04 & 0.692 \\
\hline E25 & 0.82 & 0.07 & 0.575 \\
\hline
\end{tabular}


Table 1 Average annual cumulative incidence/10,000,000 and trend of annual cumulative incidence of rare diseases in the register of the co-payment assistance policy according to KCD codes from 2011 to 2015 (Continued)

\begin{tabular}{|c|c|c|c|}
\hline $\mathrm{KCD}$ & Average annual cumulative incidence ${ }^{a}$ & Annual trend $(\beta)^{\mathrm{b}}$ & $p$-value \\
\hline E25.0 & 12.88 & 1.95 & 0.413 \\
\hline E25.8 & 0.19 & 0.06 & 0.223 \\
\hline E25.9 & 1.01 & 0.27 & 0.482 \\
\hline E27.1 & 6.64 & 0.27 & 0.766 \\
\hline E27.2 & 1.86 & 0.12 & 0.389 \\
\hline E27.4 & 121.76 & 9.85 & 0.072 \\
\hline E34.8 & 1.09 & -0.05 & 0.787 \\
\hline E55.0 & 11.05 & -1.64 & 0.290 \\
\hline E70 & 0.00 & . & . \\
\hline E70.0 & 2.17 & 0.16 & 0.726 \\
\hline E70.1 & 2.29 & 0.08 & 0.601 \\
\hline E70.2 & 0.31 & -0.22 & 0.076 \\
\hline E70.3 & 3.15 & -0.39 & 0.345 \\
\hline E70.8 & 0.04 & -0.04 & 0.182 \\
\hline E70.9 & 0.04 & 0.02 & 0.559 \\
\hline E71 & 0.23 & 0.12 & 0.559 \\
\hline E71.0 & 0.31 & 0.00 & 0.993 \\
\hline E71.1 & 2.02 & 0.18 & 0.274 \\
\hline E71.2 & 0.58 & 0.02 & 0.904 \\
\hline E71.3 & 4.00 & 0.33 & 0.437 \\
\hline E72 & 0.23 & 0.04 & 0.707 \\
\hline E72.0 & 1.79 & -0.01 & 0.945 \\
\hline E72.1 & 1.75 & 0.26 & 0.450 \\
\hline E72.2 & 2.75 & 0.66 & 0.067 \\
\hline E72.3 & 0.31 & 0.00 & 0.964 \\
\hline E72.4 & 0.27 & 0.08 & 0.340 \\
\hline E72.5 & 0.51 & -0.14 & 0.156 \\
\hline E72.8 & 0.50 & 0.09 & 0.379 \\
\hline E72.9 & 0.16 & -0.02 & 0.539 \\
\hline E73.0 & 0.00 & . & \\
\hline E73.1 & 0.04 & 0.02 & 0.559 \\
\hline E73.8 & 0.08 & 0.00 & 1.000 \\
\hline E73.9 & 0.23 & -0.04 & 0.520 \\
\hline E74 & 0.12 & 0.08 & 0.183 \\
\hline E74.0 & 4.23 & 0.81 & 0.133 \\
\hline E74.1 & 0.00 & . & . \\
\hline E74.2 & 3.08 & $-0.50^{*}$ & 0.027 \\
\hline E74.3 & 0.16 & 0.04 & 0.698 \\
\hline E74.4 & 0.16 & -0.06 & 0.314 \\
\hline E74.8 & 1.32 & -0.24 & 0.076 \\
\hline E74.9 & 0.00 & & . \\
\hline E75.0 & 0.19 & 0.00 & 0.985 \\
\hline E75.1 & 0.08 & 0.08 & 0.182 \\
\hline
\end{tabular}


Table 1 Average annual cumulative incidence/10,000,000 and trend of annual cumulative incidence of rare diseases in the register of the co-payment assistance policy according to KCD codes from 2011 to 2015 (Continued)

\begin{tabular}{|c|c|c|c|}
\hline $\mathrm{KCD}$ & Average annual cumulative incidence ${ }^{a}$ & Annual trend $(\beta)^{\mathrm{b}}$ & $p$-value \\
\hline E75.2 & 5.16 & 1.14 & 0.161 \\
\hline E75.4 & 0.23 & 0.08 & 0.315 \\
\hline E75.5 & 2.13 & $0.46^{*}$ & 0.009 \\
\hline E76 & 0.00 & . & . \\
\hline E76.0 & 0.19 & 0.04 & 0.677 \\
\hline E76.1 & 1.13 & -0.04 & 0.871 \\
\hline E76.2 & 0.62 & -0.06 & 0.672 \\
\hline E76.3 & 0.43 & 0.21 & 0.071 \\
\hline E76.8 & 0.00 & . & . \\
\hline E76.9 & 0.00 & & . \\
\hline E77 & 0.04 & 0.00 & 1.000 \\
\hline E77.0 & 0.27 & 0.12 & 0.547 \\
\hline E77.1 & 0.00 & . & . \\
\hline E77.9 & 0.00 & & . \\
\hline E79.1 & 0.23 & 0.00 & 0.995 \\
\hline E80.2 & 1.28 & -0.14 & 0.256 \\
\hline E83.0 & 16.61 & -1.24 & 0.120 \\
\hline E83.3 & 5.98 & 0.86 & 0.282 \\
\hline E84 & 0.19 & 0.13 & 0.119 \\
\hline E84.0 & 0.23 & -0.04 & 0.173 \\
\hline E84.1 & 0.08 & -0.06 & 0.057 \\
\hline E84.9 & 0.19 & -0.04 & 0.436 \\
\hline E85 & 1.98 & 0.12 & 0.685 \\
\hline E85.0 & 0.31 & 0.12 & 0.083 \\
\hline E85.2 & 0.19 & 0.15 & 0.161 \\
\hline E85.3 & 0.47 & 0.07 & 0.498 \\
\hline E85.4 & 5.00 & 0.77 & 0.084 \\
\hline E85.8 & 2.37 & 0.18 & 0.218 \\
\hline E85.9 & 16.11 & 1.49 & 0.112 \\
\hline F84.2 & 4.58 & 0.21 & 0.482 \\
\hline G10 & 5.74 & 1.14 & 0.065 \\
\hline G11 & 0.81 & 0.17 & 0.478 \\
\hline G11.0 & 0.66 & -0.22 & 0.215 \\
\hline G11.1 & 10.17 & 1.43 & 0.266 \\
\hline G11.2 & 38.45 & 5.61 & 0.051 \\
\hline G11.3 & 0.66 & -0.20 & 0.129 \\
\hline G11.4 & 10.45 & 0.60 & 0.494 \\
\hline G11.8 & 1.63 & $0.26^{*}$ & 0.030 \\
\hline G11.9 & 36.38 & 3.33 & 0.107 \\
\hline G12 & 0.97 & 0.19 & 0.189 \\
\hline G12.0 & 0.86 & 0.00 & 0.915 \\
\hline G12.1 & 9.38 & 2.32 & 0.208 \\
\hline G12.2 & 4.01 & -0.77 & 0.367 \\
\hline
\end{tabular}


Table 1 Average annual cumulative incidence/10,000,000 and trend of annual cumulative incidence of rare diseases in the register of the co-payment assistance policy according to KCD codes from 2011 to 2015 (Continued)

\begin{tabular}{|c|c|c|c|}
\hline $\mathrm{KCD}$ & Average annual cumulative incidence ${ }^{a}$ & Annual trend $(\beta)^{\mathrm{b}}$ & $p$-value \\
\hline $\mathrm{G} 12.20$ & 1.86 & 0.11 & 0.479 \\
\hline G12.21 & 55.69 & 7.32 & 0.287 \\
\hline G12.22 & 2.48 & 0.34 & 0.473 \\
\hline G12.23 & 5.83 & 0.09 & 0.905 \\
\hline G12.24 & 1.24 & 0.26 & 0.266 \\
\hline G12.8 & 3.03 & -0.27 & 0.138 \\
\hline G12.9 & 6.06 & 0.46 & 0.662 \\
\hline G13 & 0.24 & -0.20 & 0.162 \\
\hline G13.0 & 1.36 & -0.30 & 0.242 \\
\hline G13.1 & 6.87 & $4.54^{*}$ & 0.013 \\
\hline G13.2 & 0.19 & 0.04 & 0.464 \\
\hline G13.8 & 2.88 & -0.41 & 0.259 \\
\hline G20 & 2064.86 & $140.88^{*}$ & 0.029 \\
\hline G23.1 & 27.21 & -0.79 & 0.850 \\
\hline G31.81 & 1.32 & 0.21 & 0.385 \\
\hline G35 & 45.14 & -2.04 & 0.375 \\
\hline G40.4 & $24.10^{c}$ & . & . \\
\hline G40.40 & $52.65^{c}$ & . & . \\
\hline G40.41 & $33.90^{c}$ & . & . \\
\hline G41 & 8.74 & 0.18 & 0.683 \\
\hline G41.0 & 8.89 & 0.91 & 0.372 \\
\hline G41.1 & 1.32 & $0.42^{*}$ & 0.043 \\
\hline G41.2 & 13.85 & $2.08^{*}$ & 0.013 \\
\hline G41.8 & 15.68 & $2.22^{*}$ & 0.037 \\
\hline G41.9 & 124.11 & $15.76^{*}$ & 0.001 \\
\hline G51.2 & 0.19 & -0.04 & 0.436 \\
\hline G56.4 & 36.98 & $-5.16^{*}$ & 0.015 \\
\hline G60.0 & 30.34 & -2.22 & 0.166 \\
\hline G61 & 2.29 & -0.17 & 0.575 \\
\hline G61.0 & 123.72 & $12.43^{*}$ & 0.021 \\
\hline G61.1 & 0.08 & -0.02 & 0.629 \\
\hline G61.8 & 15.62 & 0.30 & 0.549 \\
\hline G61.9 & 8.50 & 0.73 & 0.408 \\
\hline G63.0 & 1.94 & 0.03 & 0.882 \\
\hline G70.0 & 137.88 & 14.27 & 0.150 \\
\hline G70.1 & 0.08 & -0.02 & 0.637 \\
\hline G70.2 & 0.08 & 0.00 & 0.996 \\
\hline G71 & 2.72 & 0.31 & 0.548 \\
\hline G71.0 & 40.04 & 2.65 & 0.567 \\
\hline G71.1 & 33.29 & 0.95 & 0.389 \\
\hline G71.2 & 6.44 & 0.82 & 0.431 \\
\hline G71.3 & 12.33 & -1.25 & 0.141 \\
\hline G71.8 & 0.81 & 0.25 & 0.288 \\
\hline
\end{tabular}


Table 1 Average annual cumulative incidence/10,000,000 and trend of annual cumulative incidence of rare diseases in the register of the co-payment assistance policy according to KCD codes from 2011 to 2015 (Continued)

\begin{tabular}{|c|c|c|c|}
\hline KCD & Average annual cumulative incidence ${ }^{a}$ & Annual trend $(\beta)^{\mathrm{b}}$ & $p$-value \\
\hline G71.9 & 2.32 & 0.88 & 0.084 \\
\hline G90.8 & 2.68 & 0.06 & 0.839 \\
\hline G95.0 & 46.81 & $-1.70^{*}$ & 0.030 \\
\hline H35.31 & 1359.61 & 103.62 & 0.121 \\
\hline H35.51 & 163.56 & 1.17 & 0.869 \\
\hline H35.58 & 6.33 & $0.78^{*}$ & 0.016 \\
\hline 127.0 & 40.80 & -3.17 & 0.204 \\
\hline 127.8 & 10.09 & 1.13 & 0.437 \\
\hline 142.0 & 521.39 & $44.36^{*}$ & 0.037 \\
\hline 142.1 & 42.31 & 4.15 & 0.139 \\
\hline 142.2 & 322.48 & 34.75 & 0.058 \\
\hline 142.3 & 1.09 & 0.11 & 0.489 \\
\hline 142.4 & 3.26 & 0.39 & 0.373 \\
\hline 142.5 & 7.10 & $0.99^{*}$ & 0.007 \\
\hline 167.5 & 261.73 & 0.38 & 0.888 \\
\hline 173.1 & 52.76 & -5.97 & 0.080 \\
\hline 178.0 & 1.40 & -0.39 & 0.203 \\
\hline 182.0 & 7.32 & -1.02 & 0.104 \\
\hline J84.0 & 4.79 & -0.86 & 0.135 \\
\hline J84.18 & 311.03 & $19.79^{*}$ & 0.006 \\
\hline K50 & 32.85 & 2.30 & 0.312 \\
\hline K50.0 & 51.57 & 6.73 & 0.262 \\
\hline K50.1 & 53.85 & 8.29 & 0.223 \\
\hline K50.8 & 35.33 & 6.61 & 0.126 \\
\hline K50.9 & 183.98 & 31.23 & 0.065 \\
\hline K51 & 52.66 & -0.32 & 0.944 \\
\hline K51.0 & 67.17 & 9.06 & 0.218 \\
\hline K51.2 & 181.53 & $27.31^{*}$ & 0.019 \\
\hline K51.3 & 54.01 & 7.81 & 0.110 \\
\hline K51.4 & 0.19 & 0.02 & 0.732 \\
\hline K51.5 & 8.14 & 2.15 & 0.099 \\
\hline K51.8 & 63.29 & 8.08 & 0.163 \\
\hline K51.9 & 373.46 & 79.92 & 0.093 \\
\hline K74.3 & 68.32 & $6.72^{*}$ & 0.008 \\
\hline K75.4 & 104.26 & $7.90^{*}$ & 0.036 \\
\hline L10.0 & 13.94 & 1.14 & 0.246 \\
\hline L10.2 & 7.18 & 0.43 & 0.286 \\
\hline L12.0 & 50.83 & $7.27^{*}$ & 0.026 \\
\hline L12.1 & 0.46 & 0.15 & 0.082 \\
\hline L12.3 & 1.52 & -0.14 & 0.555 \\
\hline M07.20 & 2.06 & $0.38^{*}$ & 0.010 \\
\hline M07.28 & 0.39 & 0.13 & 0.192 \\
\hline M08.0 & 4.58 & 0.68 & 0.575 \\
\hline
\end{tabular}


Table 1 Average annual cumulative incidence/10,000,000 and trend of annual cumulative incidence of rare diseases in the register of the co-payment assistance policy according to KCD codes from 2011 to 2015 (Continued)

\begin{tabular}{|c|c|c|c|}
\hline KCD & Average annual cumulative incidence ${ }^{a}$ & Annual trend $(\beta)^{\mathrm{b}}$ & $p$-value \\
\hline M08.1 & 0.47 & 0.11 & 0.085 \\
\hline M08.2 & 0.27 & 0.04 & 0.717 \\
\hline M08.3 & 0.39 & 0.02 & 0.881 \\
\hline M30.0 & 7.08 & -0.66 & 0.159 \\
\hline M30.1 & 9.75 & 0.66 & 0.313 \\
\hline M30.2 & 0.00 & . & \\
\hline M31.0 & 2.72 & 0.35 & 0.266 \\
\hline M31.1 & 7.26 & 0.29 & 0.370 \\
\hline M31.2 & 0.00 & . & . \\
\hline M31.3 & 8.89 & 1.02 & 0.114 \\
\hline M31.4 & 26.14 & 1.38 & 0.378 \\
\hline M31.7 & 9.26 & $2.61^{*}$ & 0.009 \\
\hline M32.1 & 13.13 & 3.76 & 0.506 \\
\hline M32.10 & 4.76 & 2.21 & 0.286 \\
\hline M32.12 & 2.48 & 0.59 & 0.333 \\
\hline M32.13 & 46.43 & 18.53 & 0.341 \\
\hline M32.15 & 20.27 & 5.24 & 0.306 \\
\hline M32.19 & 46.45 & 16.72 & 0.320 \\
\hline M32.8 & 11.48 & 1.77 & 0.506 \\
\hline M32.9 & 282.52 & 72.81 & 0.355 \\
\hline M33 & 0.66 & -0.02 & 0.858 \\
\hline M33.0 & 2.83 & 0.26 & 0.301 \\
\hline M33.1 & 22.36 & 2.58 & 0.198 \\
\hline M33.2 & 19.88 & 1.95 & 0.265 \\
\hline M33.9 & 6.25 & 0.80 & 0.485 \\
\hline M34.0 & 18.01 & 1.96 & 0.355 \\
\hline M34.1 & 3.61 & 0.35 & 0.434 \\
\hline M34.8 & 10.21 & 1.22 & 0.332 \\
\hline M34.9 & 57.97 & 7.94 & 0.379 \\
\hline M35.0 & 241.86 & $16.04^{*}$ & 0.036 \\
\hline M35.1 & 25.04 & 2.21 & 0.406 \\
\hline M35.2 & 265.39 & -14.30 & 0.221 \\
\hline M35.3 & 56.31 & $7.45^{*}$ & 0.022 \\
\hline M35.4 & 1.13 & -0.03 & 0.872 \\
\hline M35.5 & 0.08 & 0.02 & 0.646 \\
\hline M35.6 & 0.23 & -0.14 & 0.068 \\
\hline M35.7 & 0.47 & 0.06 & 0.621 \\
\hline M45 & 75.30 & 1.51 & 0.869 \\
\hline M61.1 & 0.51 & -0.16 & 0.228 \\
\hline M88 & 0.23 & 0.02 & 0.858 \\
\hline M88.0 & 0.19 & -0.04 & 0.436 \\
\hline M88.8 & 0.04 & 0.04 & 0.182 \\
\hline M88.9 & 0.16 & 0.00 & 0.992 \\
\hline
\end{tabular}


Table 1 Average annual cumulative incidence/10,000,000 and trend of annual cumulative incidence of rare diseases in the register of the co-payment assistance policy according to KCD codes from 2011 to 2015 (Continued)

\begin{tabular}{|c|c|c|c|}
\hline KCD & Average annual cumulative incidence ${ }^{a}$ & Annual trend $(\beta)^{\mathrm{b}}$ & $p$-value \\
\hline M89.0 & 13.45 & -3.51 & 0.131 \\
\hline M94.1 & 1.09 & 0.05 & 0.695 \\
\hline N25.1 & 61.63 & $-23.97^{*}$ & 0.006 \\
\hline P22.0 & 519.43 & $85.09^{*}$ & 0.001 \\
\hline Q03.1 & 4.86 & 0.01 & 0.966 \\
\hline Q04.3 & 2.60 & 0.08 & 0.732 \\
\hline Q04.6 & 8.16 & -0.14 & 0.649 \\
\hline Q05 & 1.32 & 0.07 & 0.807 \\
\hline Q05.0 & 0.12 & $-0.10^{*}$ & 0.047 \\
\hline Q05.1 & 0.04 & 0.00 & 1.000 \\
\hline Q05.2 & 0.66 & 0.23 & 0.059 \\
\hline Q05.3 & 0.23 & -0.10 & 0.273 \\
\hline Q05.4 & 0.39 & 0.04 & 0.814 \\
\hline Q05.5 & 0.12 & 0.00 & 0.984 \\
\hline Q05.6 & 0.16 & -0.06 & 0.314 \\
\hline Q05.7 & 1.75 & -0.11 & 0.477 \\
\hline Q05.8 & 1.01 & 0.03 & 0.825 \\
\hline Q05.9 & 41.94 & 3.03 & 0.163 \\
\hline Q06.2 & 0.51 & -0.08 & 0.472 \\
\hline Q07.0 & 21.11 & -0.27 & 0.718 \\
\hline Q20.0 & 1.75 & -0.07 & 0.809 \\
\hline Q20.1 & 22.26 & 0.36 & 0.548 \\
\hline Q20.2 & 0.62 & 0.15 & 0.312 \\
\hline Q20.4 & 19.02 & 5.58 & 0.127 \\
\hline Q21.8 & 3.22 & 0.17 & 0.681 \\
\hline Q22.0 & 17.12 & 1.84 & 0.086 \\
\hline Q22.6 & 1.32 & $0.32^{*}$ & 0.007 \\
\hline Q23 & 0.43 & 0.00 & 0.988 \\
\hline Q23.0 & 14.37 & $1.09 *$ & 0.039 \\
\hline Q23.1 & 96.01 & $16.07^{*}$ & 0.011 \\
\hline Q23.2 & 1.79 & 0.21 & 0.411 \\
\hline Q23.3 & 18.17 & 1.97 & 0.097 \\
\hline Q23.4 & 2.10 & 0.12 & 0.542 \\
\hline Q23.8 & 1.94 & 0.17 & 0.538 \\
\hline Q23.9 & 4.80 & 1.98 & 0.117 \\
\hline Q24.5 & 71.33 & 1.77 & 0.748 \\
\hline Q25.5 & 9.59 & 0.56 & 0.583 \\
\hline Q26.0 & 0.31 & -0.04 & 0.790 \\
\hline Q26.1 & 6.28 & $1.79 *$ & 0.001 \\
\hline Q26.2 & 12.06 & -0.85 & 0.213 \\
\hline Q26.3 & 8.14 & $1.58^{*}$ & 0.025 \\
\hline Q26.4 & 0.54 & -0.02 & 0.726 \\
\hline Q26.5 & 0.62 & -0.02 & 0.901 \\
\hline
\end{tabular}


Table 1 Average annual cumulative incidence/10,000,000 and trend of annual cumulative incidence of rare diseases in the register of the co-payment assistance policy according to KCD codes from 2011 to 2015 (Continued)

\begin{tabular}{|c|c|c|c|}
\hline $\mathrm{KCD}$ & Average annual cumulative incidence ${ }^{a}$ & Annual trend $(\beta)^{\mathrm{b}}$ & $p$-value \\
\hline Q26.6 & 0.16 & -0.02 & 0.751 \\
\hline Q38.3 & 0.08 & -0.04 & 0.305 \\
\hline Q44.2 & 13.31 & -1.30 & 0.059 \\
\hline Q64.1 & 0.66 & 0.25 & 0.180 \\
\hline Q75.1 & 2.53 & -0.40 & 0.103 \\
\hline Q75.4 & 1.94 & 0.09 & 0.283 \\
\hline Q77 & 0.12 & 0.02 & 0.777 \\
\hline Q77.0 & 0.39 & -0.02 & 0.691 \\
\hline Q77.2 & 0.12 & -0.06 & 0.354 \\
\hline Q77.3 & 1.52 & -0.09 & 0.487 \\
\hline Q77.4 & 8.98 & -0.50 & 0.107 \\
\hline Q77.5 & 0.16 & 0.00 & 0.975 \\
\hline Q77.6 & 0.04 & -0.04 & 0.182 \\
\hline Q77.7 & 2.45 & -0.19 & 0.424 \\
\hline Q77.8 & 3.19 & 0.00 & 0.995 \\
\hline Q77.9 & 0.50 & 0.07 & 0.745 \\
\hline Q78.0 & 7.54 & -0.18 & 0.793 \\
\hline Q78.1 & 4.90 & 0.13 & 0.695 \\
\hline Q78.2 & 1.79 & -0.34 & 0.158 \\
\hline Q78.4 & 5.44 & $-0.38^{*}$ & 0.004 \\
\hline Q78.5 & 0.04 & 0.04 & 0.182 \\
\hline Q78.6 & 4.98 & -0.18 & 0.846 \\
\hline Q79 & 0.20 & -0.10 & 0.109 \\
\hline Q79.0 & 8.82 & -0.03 & 0.955 \\
\hline Q79.1 & 5.94 & 0.51 & 0.354 \\
\hline Q79.2 & 3.76 & 0.50 & 0.194 \\
\hline Q79.3 & 1.83 & 0.13 & 0.640 \\
\hline Q79.4 & 0.23 & 0.04 & 0.644 \\
\hline Q79.5 & 0.43 & 0.10 & 0.288 \\
\hline Q79.6 & 1.13 & -0.14 & 0.616 \\
\hline Q79.8 & 7.07 & 0.19 & 0.609 \\
\hline Q79.9 & 5.17 & -0.23 & 0.386 \\
\hline Q81.1 & 0.04 & -0.04 & 0.182 \\
\hline Q81.2 & 1.05 & -0.20 & 0.397 \\
\hline Q85.0 & 117.58 & -12.67 & 0.080 \\
\hline Q85.1 & 17.35 & $-1.52^{*}$ & 0.050 \\
\hline Q85.8 & 14.18 & 0.50 & 0.684 \\
\hline Q86.0 & 0.04 & 0.04 & 0.182 \\
\hline Q87.0 & 8.67 & -0.69 & 0.193 \\
\hline Q87.1 & 19.00 & 0.09 & 0.912 \\
\hline Q87.2 & 8.84 & $5.42^{*}$ & 0.050 \\
\hline Q87.3 & 2.06 & -0.07 & 0.809 \\
\hline Q87.4 & 29.03 & -0.11 & 0.910 \\
\hline
\end{tabular}


Table 1 Average annual cumulative incidence/10,000,000 and trend of annual cumulative incidence of rare diseases in the register of the co-payment assistance policy according to KCD codes from 2011 to 2015 (Continued)

\begin{tabular}{|c|c|c|c|}
\hline$\overline{K C D}$ & Average annual cumulative incidence ${ }^{a}$ & Annual trend $(\beta)^{\mathrm{b}}$ & $p$-value \\
\hline Q90 & 7.10 & 0.89 & 0.293 \\
\hline Q90.0 & 5.32 & 0.59 & 0.347 \\
\hline Q90.1 & 0.23 & 0.17 & 0.058 \\
\hline Q90.2 & 0.23 & 0.11 & 0.167 \\
\hline Q90.9 & 48.67 & 6.03 & 0.278 \\
\hline Q91 & 0.00 & . & . \\
\hline Q91.0 & 0.12 & -0.08 & 0.181 \\
\hline Q91.1 & 0.00 & . & . \\
\hline Q91.2 & 0.04 & 0.00 & 1.000 \\
\hline Q91.3 & 0.70 & 0.05 & 0.583 \\
\hline Q91.4 & 0.04 & 0.04 & 0.182 \\
\hline Q91.5 & 0.08 & 0.06 & 0.058 \\
\hline Q91.7 & 0.15 & 0.06 & 0.324 \\
\hline Q93.4 & 2.14 & 0.03 & 0.946 \\
\hline Q93.5 & 10.64 & 1.05 & 0.307 \\
\hline Q96 & 3.15 & 0.06 & 0.896 \\
\hline Q96.0 & 2.13 & 0.30 & 0.533 \\
\hline Q96.1 & 0.43 & 0.17 & 0.293 \\
\hline Q96.2 & 0.04 & 0.02 & 0.559 \\
\hline Q96.3 & 1.28 & 0.26 & 0.265 \\
\hline Q96.4 & 0.54 & 0.21 & 0.105 \\
\hline Q96.8 & 3.92 & 0.16 & 0.553 \\
\hline Q96.9 & 32.59 & 5.01 & 0.216 \\
\hline Q98.0 & 11.85 & 0.64 & 0.586 \\
\hline Q98.1 & 0.62 & $0.09^{*}$ & 0.017 \\
\hline Q98.2 & 0.35 & 0.10 & 0.288 \\
\hline Q98.4 & 4.19 & 0.23 & 0.632 \\
\hline Q99.2 & 1.24 & 0.44 & 0.197 \\
\hline
\end{tabular}

Abbreviation: $K C D$ Korean Standard Classification of Diseases

${ }^{a}$ Average of annual cumulative incidence per 10,000,000 of rare diseases from 2011 to 2015 according to the KCD-7 code

blinear trend of annual cumulative incidence per 10,000,000 per year

'Average of annual cumulative incidence per 10,000,000 of rare diseases from 2014 to 2015 according to the KCD-7 code

${ }^{*} \beta$ was statistically significant $(p$ value $<0.05$ )

is a representative age-related neurodegenerative disease [6]. Several studies reported that older age was major risk factor for an increased risk of developing Parkinson's disease [25]. South Korea is undergoing unprecedented and rapid population aging and life expectancy at birth has increased steadily from 80.62 in 2011 to 82.06 in 2015 [26]. Increased longevity might increase the incidence of Parkinson's disease in South Korea. Considering the high burden and low quality of life observed with Parkinson's disease, urgent attention should be directed towards developing national support systems to alleviate the burden on patients' families [27, 28].
The number patients with sarcoidosis (D86, D86.0, D86.1, D86.2, D86.3, D86.8, D86.9) has also increased over the time. The annual cumulative incidence increased from 70.91 per $10,000,000$ in 2011 to 101.86 per 10,000,000 in 2015 (see Additional file 2). Sarcoidosis is a multisystem disorder of which the aetiology is unknown. The case-control study of 706 newly diagnosed patients conducted by National Institutes of Health found positive associations between sarcoidosis and exposures including insecticides, agricultural employment and microbial bioaerosols [29]. However, these exposures have decreased in Korea due to the shift of the 
economy from agriculture to the manufacturing and service sector. The increasing trend in the annual cumulative incidence of sarcoidosis might be explained by the increased detection rate of sarcoidosis due to the increased diagnostic techniques and frequent regular health check-ups [30-32]. The overall participation rate in primary health examinations, including the chest X-rays, has increased from 56\% in 2006 to $72 \%$ in 2013 [32]. The number of patients using computed tomography and magnetic resonance imaging, which are diagnostic tools for sarcoidosis, have also increased steadily from 4,118,434 and 631,305 in 2012 to 5,139,149 and 805,831 in 2015, respectively [33].

Upon comparison of the cumulative incidence of rare diseases in our study to the estimated incidence reported by the Orphanet [20], we observed a significant difference in the incidence of Moyamoya disease (167.5; our result: $261.73 / 10,000,000$ vs Orphanet: $3.5 / 10,000,000)$. A previous systematic review revealed that the incidence of definite Moyamoya disease in Asia (Japan, Hokkaido: 84 per 10,00,000; China: 41 per 10,00,000) was higher than in the USA (Iowa, 4 per 10,000,000) [34]. Although Asian countries have a predilection for developing Moyamoya disease, the cumulative incidence of Moyamoya disease in South Korea was higher than in other Asian countries. This high incidence among South Koreans has also been supported by the findings from other studies which have used the medical claim data of NHIS (average annual cumulative incidence 2005-2013: 270/10,000,000) [35]. The high incidence of Moyamoya disease in East Asia might be partly explained by genetic effects of developing the rare disease [36]. A previous study showed a strong association between the p.R4810K mutation in RNF213 [24], which has a relatively high prevalence among East Asians and is a risk factor for the development of Moyamoya disease [37].

To the best of our knowledge, this is the first study to estimate the cumulative incidence of an entire spectrum of rare diseases in Korea by using data from a nationwide administrative database in the register of rare diseases. However, our results should be interpreted within the context of the study's limitations. First, only patients with rare diseases who were supported by the government were included in our study. Considering the complexity of the diagnosis of rare diseases, there is a possibility that many patients with rare diseases did not receive a definite diagnosis by the physician, which is a prerequisite for national support for rare diseases. Furthermore, the method of grouping patients with rare diseases according to the ICD codes could overestimate the actual incidence of rare diseases when the specificity of the ICD coding was not validated [38]. Therefore, our estimated annual cumulative incidence might not reflect the actual incidence of rare diseases. However, our results do supplement the disadvantages observed in other studies which have used the ICD codes in medical claim data leading to the ambiguity of the diagnosis of rare diseases. Our study included patients who met the diagnostic criteria on the basis of imaging studies, biochemistry, immunology, smear, culture test, histological examination and clinical diagnosis for benefiting from the national support. Second, our study could not include all rare diseases for which the prevalence was less than 20,000 patients in South Korea. Particularly, our result could not show the annual cumulative incidence of almost all cases of cancer because the medical expenses were covered by other co-payment assistance policies established by the NHIS. Third, owing to the available options for KCD codes on the application form of the register of the co-payment assistance policy for rare and incurable diseases, multiple diseases with similar characteristics were included under one KCD code. We could not separately analyse each rare disease included under one KCD code using these administrative data. Fourth, our study used the year of definite diagnosis by the physician in the application form as the year of disease occurrence. Because the definite diagnosis of a disease is usually confirmed after the disease progresses enough to affect the quality of life of patients [11], the year of disease onset in our study might not represent the actual incident date of the rare disease. Fifth, the total number of rare diseases increased abruptly from 2013 to 2014 after the Ministry of Health and Welfare of Korea adopted the agenda for expanding the health coverage for rare and incurable diseases. The number of patients enrolled in the register of the co-payment assistance policy was not constant and varied based on government policy, which might have therefore disrupted the calculation of the actual incidence of the rare diseases. For yearly comparisons of the incidence of each rare disease, the annual cumulative incidence of each rare disease is shown in Additional file 2. Sixth, no cases of 18 (4.34\%) KCD code diseases (D56, D81, D82, D83, D84.0, E70, E73.0, E74.1, E74.9, E76, E76.8, E76.9, E77.1, E77.9, M30.2, M31.2, Q91, Q91.1) were reported during the study period. Given that the NHIS determined the target disease of the co-payment policy before 2011, it is possible that there were no cases of registrants with these KCD codes for 2011-2015. Seventh, in order to calculate the incidence of rare disease, the denominator should be limited to the "population at risk" for developing rare disease. However, we set the denominator as the number of residents with health insurance coverage in each year. Additional studies are needed to better understand the epidemiology of each rare disease by using administrative records of rare disease registries in Korea.

\section{Conclusion}

We analysed the average annual cumulative incidence of rare diseases and the trends in the annual cumulative incidence per year from 2011 to 2015 in Korea by using 
data from the nationwide administrative database complied by the Korean NHIS. The number of rare diseases showing an increasing trend in annual cumulative incidence was higher than the number of diseases showing a decreasing trend in annual cumulative incidence. Although we calculated the annual cumulative incidence of all the rare diseases documented in Korea, it is difficult to evaluate the precise epidemiology of these rare diseases considering that the definition and diagnosis varies by country and considering the difficulty with detecting valid cases. Further detection strategies are needed to establish the incidence of each rare disease, considering the importance of establishing a health policy based on the actual incidence of targeted diseases.

\section{Additional files}

Additional file 1: Table S1. Targeted rare diseases included in the co-payment assistance policy established by the NHIS according to KCD codes in Korea. Description of data: Additional file 1 includes the full list of targeted rare diseases covered by the co-payment assistance policy for rare and incurable diseases in South Korea. Registered patients with rare diseases make out-of-pocket payments that comprise about $10 \%$ of the total cost of medical treatment, which is normally $20 \%-60 \%$ of the total cost of treatment. (DOCX $48 \mathrm{~kb}$ )

Additional file 2: Table S2. Annual cumulative incidence per 10,000, 000 insured population for rare diseases in the register of the co-payment assistance policy according to KCD codes from 2011-2015. Description of data: Additional file 2 includes the annual cumulative incidence per $10,000,000$ for each targeted rare disease from 2011-2015. Annual cumulative incidence per 10,000,000 was calculated as the total number of newly enrolled patients with the KCD-7 code in the register of the copayment assistance policy for rare and incurable diseases during a calendar year, divided by the number of residents with health insurance coverage in each year. (DOCX $80 \mathrm{~kb})$

\section{Abbreviations}

ICD: International Classification of Diseases; KCD: Korean standard classification of diseases; NHI: National Health Insurance; NHIS: National Health Insurance Service

\section{Acknowledgments}

We acknowledge the government authorities of the Department of Big Data Steering, National Health Insurance Service. We thank all the scientists working in the National Health Insurance Service who collected and handled the data from the register of the co-payment assistance policy for rare and incurable diseases.

\section{Funding}

This work was supported by the National Health Insurance Service. The funding source had no role in the study design, analysis, decision to publish, or preparation of the manuscript.

\section{Availability of data and materials}

This study used National Health Information Database data (No. NHIS-2017-1244) complied by the NHIS. The dataset supporting the conclusions of this article is not publicly available owing to the restrictive policy of the National Health Insurance Sharing Service (https://nhiss.nhis.or.kr/bd/ay/bdaya001iv.do) as an affiliate of the NHIS, but are available from the corresponding author on reasonable request.

\section{Authors' contributions}

SSL and JHY conceptualized and designed the study. JHP and BRP collected and handled the raw data from the register of the co-payment assistance policy for rare and incurable diseases. SSL, WL, and YKK performed the formal analysis. SSL, JK, YKK, and WL wrote the original draft of the manuscript. JHY devised and supervised the entire process. JHY and WL validated the study. All of the authors read and approved the final manuscript.

\section{Ethics approval and consent to participate}

This study was approved by the Institutional Review Board (IRB) of Severance Hospital, Seoul, South Korea (IRB approval number: Y-2017-0013). As this was a retrospective study, the IRB waived the requirement for informed consent.

\section{Consent for publication}

Not applicable.

\section{Competing interests}

The authors declare that they have no competing interests.

\section{Publisher's Note}

Springer Nature remains neutral with regard to jurisdictional claims in published maps and institutional affiliations.

\section{Author details}

${ }_{1}^{1}$ Graduate School of Public Health, Yonsei University, Seoul, Republic of Korea. ${ }^{2}$ The Institute for Occupational Health, Yonsei University College of Medicine, Seoul, Republic of Korea. ${ }^{3}$ Department of Occupational and Environmental Medicine, Seoul St. Mary's Hospital, College of Medicine, The Catholic University of Korea, Seoul, South Korea. ${ }^{4}$ Department of Big Data Steering, National Health Insurance Service, Seoul, South Korea. ${ }^{5}$ Department of Preventive Medicine and Public Health, Yonsei University College of Medicine, Seoul, Republic of Korea.

Received: 13 February 2018 Accepted: 11 February 2019

Published online: 18 February 2019

\section{References}

1. European Organisation for Rare Diseases (EURORDIS). Rare diseases: understanding this public health priority. Paris: European Organisation for Rare Diseases; 2005. Available at https://www.eurordis.org/IMG/pdf/ princeps_document-EN.pdf. Accessed 12 Sep 2018

2. Field $M$, Boat T. Institute of medicine (us) committee on accelerating rare diseases research and orphan product development. Rare diseases and orphan products: accelerating research and development; 2010.

3. NHISS. The statistics of national heatlh insurance: population with health insurance coverage. http://nhiss.nhis.or.kr/bd/ad/bdada013cv.do. Accessed 13 Jan 2018.

4. Angelis A, Tordrup D, Kanavos P. Socio-economic burden of rare diseases: a systematic review of cost of illness evidence. Health Policy. 2015;119(7):964-79.

5. Commission of the European Communities. Communication from the commission to the council, the european parliament, the european economic and social committee, and the committee of the regions: on rare diseases: Europe's challenges. 2008.

6. Franceschi C, Garagnani P, Morsiani C, Conte M, Santoro A, Grignolio A, et al. The continuum of aging and age-related diseases: common mechanisms but different rates. Front Med (Lausanne). 2018;5:61.

7. Feltmate K, Janiszewski PM, Gingerich S, Cloutier M. Delayed access to treatments for rare diseases: Who's to blame? Respirology. 2015;20(3):361-9.

8. Rode J. Rare diseases: understanding this public health priority; 2005.

9. Gahl WA, Markello TC, Toro C, Fajardo KF, Sincan M, Gill F, et al. The national institutes of health undiagnosed diseases program: insights into rare diseases. Genet Med. 2011;14(1):51-9.

10. Anderson M, Elliott EJ, Zurynski YA. Australian families living with rare disease: experiences of diagnosis, health services use and needs for psychosocial support. Orphanet J Rare Dis. 2013;8:22.

11. Schieppati A, Henter JI, Daina E, Aperia A. Why rare diseases are an important medical and social issue. Lancet. 2008;371(9629):2039-41.

12. Hesselgrave BL. Helping to manage the high cost of rare diseases. Manag Care Q. 2003;11(1):1-6.

13. Schey C, Milanova T, Hutchings A. Estimating the budget impact of orphan medicines in Europe: 2010 - 2020. Orphanet J Rare Dis. 2011;6:62.

14. Shafie AA, Chaiyakunapruk N, Supian A, Lim J, Zafra M, Hassali MA. State of rare disease management in Southeast Asia. Orphanet J Rare Dis. 2016;11(1):107. 
15. Gauld R, Ikegami N, Barr MD, Chiang TL, Gould D, Kwon S. Advanced Asia's health systems in comparison. Health Policy. 2006;79(2-3):325-36.

16. Ferrari A, Bisogno G, De Salvo GL, Indolfi P, Perilongo G, Cecchetto G. The challenge of very rare tumours in childhood: the Italian TREP project. Eur J Cancer. 2007:43(4):654-9.

17. Seo SR, Jang SY, Lee GY, Choi B, Chun H, Cho EJ, et al. Prevalence of amyloidosis in Korea. Orphanet J Rare Dis. 2017;12(1):152.

18. Ryder S, Leadley RM, Armstrong N, Westwood M, de Kock S, Butt T, et al. The burden, epidemiology, costs and treatment for Duchenne muscular dystrophy: an evidence review. Orphanet J Rare Dis. 2017;12(1):79.

19. Groth KA, Hove H, Kyhl K, Folkestad L, Gaustadnes M, Vejlstrup N, et al. Prevalence, incidence, and age at diagnosis in Marfan syndrome. Orphanet J Rare Dis. 2015;10:153.

20. Orphanet. Orphanet Report Series: Prevalence and incidence of rare diseases; 2018. Available at https://www.orpha.net/consor/cgi-bin/Education. php?!ng=EN. Accessed 12 Sept 2018.

21. Lacaze $\mathrm{P}$, Millis N, Fookes M, Zurynski $Y$, Jaffe A, Bellgard $M$, et al. Rare disease registries: a call to action. Intern Med J. 2017;47(9):1075-9.

22. Korea S. Korean standard classification of diseases. Seoul: Statistics Korea; 2010.

23. WHO. International Statistical Classification of Diseases and Related Health Problems. 10th Revision. Geneva: World Health Organisation; 2004. Available at http://apps.who.int/classifications/icd10/browse/2016/ en. Accessed 12 Sep 2018

24. Ma J, Liu Y, Ma L, Huang S, Li H, You C. RNF213 polymorphism and Moyamoya disease: A systematic review and meta-analysis. Neurol India. 2013;61(1):35-9.

25. Kieburtz K, Wunderle KB. Parkinson's disease: evidence for environmental risk factors. Mov Disord. 2013;28(1):8-13.

26. Statistcs Korea, Vital Statiscs. Life expectancy at birth-total (age): KOSIS. Available at http://kosis.kr/statHtml/statHtml.do?orgld=101\&tblld=DT_ 1B8000F\&conn_path=I2\&language=en. Accessed 30 Aug 2018

27. Schmotz C, Richinger C, Lorenzl S. High burden and depression among latestage idiopathic Parkinson disease and progressive Supranuclear palsy caregivers. J Geriatr Psychiatry Neurol. 2017;30(5):267-72.

28. Bohingamu Mudiyanselage S, Watts JJ, Abimanyi-Ochom J, Lane L, Murphy AT, Morris ME, et al. Cost of living with Parkinson's disease over 12 months in Australia: a prospective cohort study. Parkinsons Dis. 2017;2017:5932675.

29. Newman LS, Rose CS, Bresnitz EA, Rossman MD, Barnard J, Frederick M, et al. A case control etiologic study of sarcoidosis: environmental and occupational risk factors. Am J Respir Crit Care Med. 2004;170(12):1324-30

30. Oh HY, Kim EY, Kim J-E, Kim YJ, Choi H-Y, Cho J, et al. Trends of CT use in the pediatric emergency Department in a Tertiary Academic Hospital of Korea during 2001-2010. Korean J Radiol. 2012;13(6):771-5.

31. Oh HY, Kim EY, Cho J, Yang HJ, Kim JH, Kim HS, et al. Trends of CT use in the adult emergency Department in a Tertiary Academic Hospital of Korea during 2001-2010. Korean J Radiol. 2012;13(5):536-40.

32. Kang YJ, Myong J-P, Eom H, Choi B, Park JH, Kim L. E-A. The current condition of the workers' general health examination in South Korea: a retrospective study. Ann Occup Environ Med. 2017;29(1):6.

33. Health Insurance Review \& Assessment Service. Healthcare Bigdata Hub. Medical practice (Examination/Surgery, etc.) Statistics. Health Insurance Review \& Assessment Service. Available at http://opendata.hira.or.kr/op/opc/ olapDiagBhvInfo.do\#none. Accessed 28 Aug 2018.

34. Kleinloog R, Regli L, Rinkel GJ, Klijn CJ. Regional differences in incidence and patient characteristics of moyamoya disease: a systematic review. J Neurol Neurosurg Psychiatry. 2012;83(5):531-6.

35. Kim T, Lee H, Bang JS, Kwon OK, Hwang G, Oh CW. Epidemiology of Moyamoya disease in Korea: based on National Health Insurance Service data. J Korean Neurosurg Soc. 2015;57(6):390-5.

36. Hunter DJ. Gene-environment interactions in human diseases. Nat Rev Genet. 2005;6(4):287-98.

37. Liu W, Hitomi T, Kobayashi H, Harada KH, Koizumi A. Distribution of moyamoya disease susceptibility polymorphism p.R4810K in RNF213 in east and southeast Asian populations. Neurol Med Chir (Tokyo). 2012; 52(5):299-303

38. O'Malley KJ, Cook KF, Price MD, Wildes KR, Hurdle JF, Ashton CM. Measuring Diagnoses: ICD Code Accuracy. Health Serv Res. 2005:40(5 Pt 2):1620-39.

Ready to submit your research? Choose BMC and benefit from:

- fast, convenient online submission

- thorough peer review by experienced researchers in your field

- rapid publication on acceptance

- support for research data, including large and complex data types

- gold Open Access which fosters wider collaboration and increased citations

- maximum visibility for your research: over $100 \mathrm{M}$ website views per year

At BMC, research is always in progress.

Learn more biomedcentral.com/submissions 